\title{
PREVALÊNCIA DE SINTOMAS URINÁRIOS NO TERCEIRO TRIMESTRE DA GESTAÇÃO
}

\author{
Katia Pary Scarpa, Viviane Herrmann*, Paulo César Rodrigues Palma, Cássio luiz Zanettini Ricetto, Sirlei Morais
}

Trabalho realizado na Universidade Estadual de Campinas - SP

\author{
*Correspondência: \\ Rua Alexandre Fleming, \\ 101, Cidade Universitária \\ Zeferino Vaz \\ Caixa Postal: 6081 \\ CEP: 13083-970, \\ Campinas, São Paulo, \\ (19) 3788-9306 \\ vherrmann@uol.com.br
}

\begin{abstract}
RESUMO
OBjEtivo. 0 objetivo deste estudo foi avaliar a prevalência de sintomas urinários irritativos no terceiro trimestre da gestação e a correlação destes com a paridade e a forma de término de partos anteriores.

Métodos. Foram selecionadas 340 gestantes no terceiro trimestre da gestação, atendidas no Ambulatório de Pré-Natal do Hospital das Clínicas da Unicamp, entre junho e outubro de 2003. O desenho consistiu em um estudo analítico de corte transversal.
\end{abstract}

Resultados. Os sintomas mais prevalentes foram: noctúria (80,6\%); polaciúria (70,3\%) e urgência miccional (44,4\%). Embora não houvesse associação entre a prevalência de sintomas irritativos e a forma de término dos partos anteriores, observou-se correlação significativa entre multiparidade e os sintomas de enurese noturna, noctúria e frequência urinária. Conclusão. Concluiu-se que, no grupo estudado, a gestação per se foi um fator de risco para a ocorrência de sintomas urinários irritativos.

UnIteRmos: Sintomas urinários irritativos. Gestação. Paridade. Forma de término do parto.

\section{INTRODUÇÃo}

Os sintomas urinários irritativos são considerados problemas comuns que afetam principalmente as mulheres, podendo causar constrangimento e restrição das atividades diárias, influindo de forma negativa na qualidade de vida da mulher. Na literatura, encontra-se poucos estudos epidemiológicos descritivos sobre a prevalência de sintomas urinários irritativos em determinadas populações!.

A ocorrência de sintomas urinários geralmente está associada a alterações anatômicas e a danos neurológicos decorrentes do trauma obstétrico, que podem levar à importante perda de sustentação do colo vesical e da uretra proximal ${ }^{1,2}$. Entretanto, estudos relatam o aparecimento destes sintomas no decorrer da primeira gestação $0^{3,4}$, sugerindo fortemente que os fatores envolvidos em seu desencadeamento não se encontram exclusivamente relacionados aos danos provocados pelo parto vaginal traumático. Durante a gestação, modificações anatômicas e funcionais no trato urinário inferior possivelmente alteram os mecanismos envolvidos com a continência urinária, desencadeando sintomas urinários ${ }^{5,6,7}$. Chaliha et al. ${ }^{3}$, analisando 549 gestantes nulíparas, observaram que $81,1 \%$ apresentaram polaciúria, $67 \%$ noctúria e $22,9 \%$ urgência miccional. A gestação per se parece envolver processos que predispõem a recorrência de sintomas urinários nas gestações subseqüentes e, posteriormente, ao longo da vida ${ }^{8}$.

$\mathrm{Na}$ literatura, a exata natureza dos fatores de risco relacionados ao surgimento de sintomas urinários durante a gestação não é totalmente compreendida. Pretendemos, neste estudo, avaliar a prevalência de sintomas urinários irritativos no terceiro trimestre da gestação e sua relação com a forma de término de partos anteriores e a paridade. Futuramente, o reconhecimento destes fatores poderá contribuir para a elaboração de programas preventivos que visem melhorar a qualidade da atenção oferecida durante o pré-natal pelos profissionais da saúde.

\section{Métodos}

Participaram do estudo 340 gestantes com idade gestacional $\geq 26$ semanas, selecionadas do Ambulatório de Pré-Natal do Departamento de Tocoginecologia, no Hospital das Clínicas da Faculdade de Ciências Médicas da Unicamp, entre junho e outubro de 2003, após assinatura do Termo de Consentimento Livre e Esclarecido. Este estudo foi aprovado pelo Comitê de Ética em Pesquisa desta instituição. Um questionário pré-testado foi aplicado individualmente às gestantes pela fisioterapeuta responsável pelo estudo. Foram registradas informações relativas às características demográficas das pacientes, dados obstétricos (forma de término de partos anteriores e paridade), assim como a presença de sintomas urinários irritativos na gestação atual.

Os critérios de exclusão foram: diabetes mellitus; litíase renal; doença pulmonar obstrutiva crônica; infecção do trato urinário (ITU); história pregressa de cirurgia pélvica; e uso de medicações que interferem na função do trato urinário inferior (TUI).

Os sintomas urinários irritativos foram definidos segundo o subcomitê de padronização da Sociedade Internacional de Continência?: noctúria: acordar uma ou mais vezes à noite para urinar; polaciúria: urinar com muita freqüência durante o dia; urgência: desejo súbito e incontrolável de urinar, difícil de ser adiado; urge-incontinência: perda involuntária de urina acompanhada ou imediatamente precedida por urgência; enurese noturna: perda urinária que ocorre durante o sono.

Os resultados foram analisados por meio do cálculo de freqüências, teste de Qui-quadrado ou Exato de Fisher, quando necessário, e do cálculo de razão de prevalência (RP). O nível de significância foi assumido em $5 \%$. 
Tabela I - Prevalência de sintomas urinários irritativos no terceiro trimestre da gestação $(n=340)$

\begin{tabular}{|c|c|c|}
\hline Sintomas urinários irritativos & $n$ & $\%$ \\
\hline Noctúria & 274 & 80,6 \\
\hline Polaciúria & 239 & 70,3 \\
\hline Urgência & $|5|$ & 44,4 \\
\hline Urge-incontinência & 77 & 22,6 \\
\hline Enurese noturna & 15 & 4,4 \\
\hline
\end{tabular}

Tabela 2 - Distribuição percentual de sintomas urinários irritativos segundo a paridade $(n=340)$

\begin{tabular}{|c|c|c|c|c|c|c|c|c|c|}
\hline & \multicolumn{2}{|c|}{ Nulíparas } & \multicolumn{2}{|c|}{ Primíparas } & \multicolumn{2}{|c|}{ Multíparas $\leq 3$} & \multicolumn{2}{|c|}{ Multíparas $\geq 4$} & \multirow[b]{2}{*}{ Valor-p } \\
\hline & $\mathrm{n}$ & $\%$ & $\bar{n}$ & $\%$ & $n$ & $\%$ & $\bar{n}$ & $\%$ & \\
\hline Noctúria & $10 \mid$ & 77,7 & 77 & 80,2 & 71 & 79,8 & 25 & 100,0 & $0,0354 *$ \\
\hline Polaciúria & 85 & 65,4 & 71 & 74,0 & 60 & 67,4 & 23 & 92,0 & $0,0327 *$ \\
\hline Urgência & 61 & 46,9 & 38 & 39,6 & 39 & 43,8 & 13 & 52,0 & 0,6075 \\
\hline Urge-incontinência & 25 & 19,2 & 21 & 21,9 & 24 & 27,0 & 7 & 28,0 & 0,5211 \\
\hline Enurese noturna & 4 & 3,1 & 3 & 3,1 & 4 & 4,5 & 4 & 16,0 & $0,0665 *$ \\
\hline
\end{tabular}

Teste Qui-quadrado

*Teste Exato de Fisher

Tabela 3 - Distribuição percentual entre sintomas urinários irritativos e a forma de término do parto, exclusivamente vaginal $(n=I I I)$ ou exclusivamente cesáreo $(n=68)($ Total $n=\mid 79)$

\begin{tabular}{|c|c|c|c|c|c|c|c|}
\hline \multirow{7}{*}{$\begin{array}{l}\text { Noctúria } \\
\text { Polaciúria } \\
\text { Urgência } \\
\text { Urge-incontinência } \\
\text { Enuresenoturna }\end{array}$} & \multicolumn{2}{|c|}{ Cesáreo } & \multicolumn{5}{|c|}{ Vaginal } \\
\hline & $\bar{n}$ & $\%$ & $n$ & $\%$ & & IC 95\%) & Valor-p \\
\hline & 53 & 77,9 & 91 & 82,0 & 1,05 & $(0,90$ a l, 23) & 0,5082 \\
\hline & 49 & 72,1 & 80 & 72,1 & 1,00 & $(0,83$ a l, 21$)$ & 0,9985 \\
\hline & 28 & 41,2 & 46 & 41,4 & $1,0 \mid$ & $(0,70$ a l 1,44$)$ & 0,9722 \\
\hline & 15 & 22,1 & 27 & 24,3 & 1,10 & $(0,63$ a 1,92$)$ & 0,7285 \\
\hline & 3 & 4,4 & 3 & 2,7 & 0,61 & $(0,13$ a 2,95$)$ & $0,5375 *$ \\
\hline
\end{tabular}

Teste Qui-quadrado

*Teste Exato de Fisher

\section{Resultados}

A idade das 340 gestantes estudadas variou de 17 a 46 anos, sendo a idade média 26,4 anos. Com relação à etnia, 47,1\% se autoclassificaram brancas, 41 ,2\% pardas e 10,9\% negras. No momento da entrevista, 47\% viviam em união consensual, 34,7\% eram casadas e 15,6\% eram solteiras. Quanto à escolaridade, a maioria não completou o ensino fundamental.

Das 340 gestantes, 94,4\% apresentaram um ou mais sintomas urinários irritativos. Os sintomas mais freqüentemente relatados foram noctúria, polaciúria e urgência, conforme dados apresentados na Tabela I.

Com relação à paridade, as gestantes foram divididas em quatro grupos: 38,2\% nulíparas, 28,2\% primíparas, 26,2\% multíparas com até três partos e 7,4\% multíparas com quatro partos ou mais. As multíparas com quatro partos ou mais apresentaram 100\% e 92\% de noctúria e polaciúria, respectivamente. $\bigcirc$ estudo da associação entre a presença de sintomas urinários irritativos e a paridade evidenciou diferença estatisticamente significativa entre noctúria $(p=0,0354)$ e polaciúria
( $p=0,0327$ ) quando comparadas as multíparas com quatro partos ou mais aos outros grupos. Também foi elevada a prevalência destes sintomas entre as nulíparas, 77,7\% e 65,4\%, respectivamente, mostrando o risco do desencadeamento destes sintomas na primeira gestação (Tabela 2). A análise da razão de prevalência (IC 95\%) mostrou que as multíparas (=4) apresentaram I,29 e I,4l vez mais chances de desencadear noctúria e polaciúria, respectivamente, e 5,2 vezes mais chances de desencadear enurese noturna quando comparadas às nulíparas.

Com relação à forma de término de partos anteriores, as gestantes foram selecionadas por parto exclusivamente vaginal ou exclusivamente cesáreo. A associação entre sintomas urinários irritativos e a forma de término do parto não demonstrou diferença estatisticamente significativa (Tabela 3).

\section{Discussão}

presente estudo evidenciou uma alta prevalência de sintomas urinários irritativos no terceiro trimestre da gestação, podendo este 
fato estar associado à pressão exercida pela cabeça fetal sobre a bexiga com conseqüente redução da capacidade vesical ${ }^{5}$. Os sintomas mais freqüentemente observados foram a noctúria e polaciúria em 80,6\% e $70,3 \%$ dos casos, respectivamente, sendo estes resultados semelhantes aos observados por outros autores ${ }^{6,10}$. Entretanto, sintomas de urgência e particularmente de urge-incontinência tiveram uma prevalência acentuadamente maior do que a observada por outros autores ${ }^{3,6}$. É provável que diferenças na argüição das pacientes ou até mesmo na definição deste sintoma reflitam esta disparidade.

Acredita-se que a variação dos níveis hormonais, a qual ocorre paralelamente ao desenvolvimento da gestação, possa influenciar o mecanismo de continência ${ }^{3,5,11}$. Wijma et al.1'12 observaram que altos níveis de progesterona levam à hipotonicidade das estruturas do assoalho pélvico, influenciando o desencadeamento de sintomas urinários no início da gestação. O'Boyle et al..$^{13}$ observaram aumento significativo da mobilidade uretral em gestantes nulíparas, sugerindo que alterações fisiológicas no assoalho pélvico podem ser objetivamente demonstradas antes do parto.

Altos níveis de hormônio relaxina estão presentes na circulação sangüínea durante a gestação, atingindo maiores níveis no terceiro trimestre ${ }^{15}$. O aumento na mobilidade articular, ocorrido em gestantes nulíparas, sugere que também o relaxamento nas estruturas do tecido conjuntivo da pelve, neste período, predispõe o desencadeamento de sintomas urinários ${ }^{16}$. Para Ostgaard et al. ${ }^{17}$, as gestantes que apresentaram maior grau de frouxidão articular foram as que desenvolveram maior diâmetro abdominal, provavelmente pelo aumento da elasticidade da parede abdominal resultante da diminuição da produção de colágeno causada pela ação do hormônio relaxina. As alterações posturais que ocorrem ao longo da gestação possivelmente estão relacionadas aos altos níveis de relaxina, que influenciam a estrutura músculo-esquelética ${ }^{18,19}$. Para Sapsford \& Hodges $^{20}$, quando a parede abdominal encontra-se relaxada, há uma diminuição na atividade eletromiográfica da musculatura do assoalho pélvico, com conseqüente diminuição da função de sustentação do assoalho pélvico, comprometendo o mecanismo da continência urinária.

A gestação per se parece envolver processos que predispõem a recorrência de incontinência em gestações subseqüentes e ao longo da vida $^{8}$, mas alterações patológicas resultantes de danos teciduais no parto podem ser sobrepostas ${ }^{7}$. Neste estudo, a paridade (multíparas = 4) foi significativamente associada ao aumento da prevalência de sintomas na gestação. $O$ estiramento acentuado dos elementos de sustentação do assoalho pélvico durante o período expulsivo aumenta a probabilidade de danos a estas estruturas ${ }^{14,21}$. O primeiro parto vaginal é responsável por relaxamento tecidual significativo das estruturas do assoalho pélvico e dano nervoso ${ }^{22}$, sendo que os partos subseqüentes elevam apenas discretamente a prevalência.

Assim como observado por outros autores ${ }^{2,11}$, o presente estudo não mostrou associaçãa entre a presença de sintomas urinários irritativos na gestação e a forma de término de partos anteriores. Pessina et al. ${ }^{23}$ propõem que sintomas irritativos possam resultar de isquemia do músculo detrusor associada à parcial denervação, desencadeando um comportamento contrátil anormal. Freqüentemente, estes sintomas persistem ao longo da vida, interferindo intensamente nas atividades diárias da mulher e comprometendo gravemente sua qualidade de vida.
Segundo Morkved et al. ${ }^{24}$, exercícios da musculatura do assoalho pélvico em gestantes nulíparas preveniram o desencadeamento de sintomas urinários na gestação e após o parto. O conhecimento de uma alta prevalência de sintomas urinários na gestação pode trazer um argumento favorável para a implementação de uma intervenção preventiva na gestação, como um programa intensivo de exercícios da musculatura do assoalho pélvico que mantenha a função satisfatória do mecanismo da continência e também a função sexual da mulher.

\section{Conflito de interesse: não há}

\section{SUMMARY}

\section{Prevalence of URINARY SyMPtoms IN the thiRd tRIMester of PREGNANCY}

BACKGROUND. This study evaluated the prevalence of irritative bladder symptoms of women in the third trimester of pregnancy and the correlation to parity and route of delivery.

METHODS. Between/une and October2003, 340 women attendingthe prenatal clinic at the Department of Obstetrics and Gynecology, State University of Campinas (Unicamp) were selected for study.

RESULTS. On the total, $80.6 \%$ presented nocturia, $70.3 \%$ presented urinary frequency and $44.4 \%$ presented urgency. No statistic correlation was observed between irritative bladder symptoms and route of delivery however, when considering parity, nocturia and urinary frequency were significantly more frequent in multiparous women.

CONCLUSION. In the population under study pregnancy per se was associated to a high prevalence of irritative bladder symptoms. [Rev Assoc Med Bras 2006; 52(3): I53-6]

KEY wORDS: Irritative bladder symptoms. Pregnancy. Parity. Route of delivery.

\section{REFERÊNCIAS}

I. Hunskaar S, Arnold EP, Burgio KL, Diokno AC, Herzog AR, Mallett VT Epidemiology and natural history of urinary incontinence. Int Urogynecol 12000; 11:301-319.

2. Chaliha C, Khullar V, Stanton SL, Monga A, Sultan AH. Urinary symptoms in pregnancy: are they useful for diagnosis? Br J Obstet Gynecol 2002; 109:1 181-1183

3. Chaliha C, Kalia V, Stanton SL, Monga ASH, Sultan AH. Antenatal prediction of postpartum urinary and fecal incontinence. Obstet Gynecol 1999; 94:68-694.

4. MacLennan AH, Taylor AW, Wilson DH, Wilson D. The prevalence of pelvic disorders and their relationship to gender, age, parity and mode of delivery. Br J Obstet Gynecol 2000; 107: | 460 - I 470

5. Thorp JMJr, Norton PA, Wall LL, Kuller JA, Eucker B, Wells E. Urinary incontinence in pregnancy and the puerperium: a prospective study. Am J Obstet Gynecol 1999; 181:266-273.

6. Nel JT, Diedericks A, Joubert G, Arndt K. A prospective clinical and urodynamic study of bladder function during and after pregnancy. Int Urogynecol J 200 I ; 12:21-26.

7. Chaliha C, Stanton SL. Urological problems in pregnancy. BJU Int 2002; 89:469-476

8. Viktrup L, Lose G. Lower urinary tract symptoms 5 years after the first delivery. Int Urogynecol I 2000; I 1:336-340.

9. Abrams P. Cardozo I, Fall M, Griffiths D, Rosier P. Ulmsten $U$ et al. The standardization of terminology of lower urinary tract function: report from standardization sub committee of the International Continence Society. Neurourol Urodyn 2002; 21:167-178. 
10. Aslan D, Aslan G, Yamazhan M, Ispahi C, Tinar S. Voiding symptoms in pregnancy: An assessment with International Prostate Symptom Score. Gynecol Obstet Invest 2003; 55:46-49.

I I. Hvidman L, Foldspang A, Mommsen S, Bugge Nielsen J. Correlates to urinary incontinence in pregnancy. Int Urogynecol ] 2002; | 3:278-283.

12. Wijma J, Potters AEW, Wolf BTHM, Tinga DJ, Aarnoudse JG. Anatomical and functional changes in the lower urinary tract during pregnancy. $\mathrm{Br}$ J Obstet Gynaecol 200 I; 1 08:726-732.

13. O'Boyle AL, O'Boyle JD, Ricks RE, Patience TH, Calhoun B, Davis G. The natural history of pelvic organ support during pregnancy. Int Urogynecol J 2003; 14:46-49.

14. Van Geelen JM, Lemmens WAJG, Eskes TKAB, Martin CB. The urethral pressure profile in pregnancy and after delivery in healthy nulliparous women. Am J Obstet Gynecol 1982; 144:636-649.

15. MacLennan AH, Nicolson R, Green RC, Bath M. Serum relaxin and pelvic pain of pregnancy. Lancet 1986; 243-245.

16. Tincello DG, Adams EJ, Richmond DH. Antenatal screening for postpartum urinary incontinence in nulliparous women: a pilot study. Eur J Obstet Gynecol Reprod Biol 2002; 101:70-73.

17. Östgaard HC, Andersson GBJ, Schultz AB, Miller JAA. Influence of some biomechanical factors on low-back pain in pregnancy. Spine 1993; 18:61-65.

18. Nicholls JA, Grieve DW. Posture, performance and discomfort in pregnancy. Ergonomics 1992; 23: 128-132.
19. Franklin ME, Conner-Kerr T. An analysis of posture and back pain in the first and third trimesters of pregnancy. J Orthop Sports Phys Ther 1998; 28:133-138.

20. Sapsford RR, Hodges PW. Contraction of the pelvic floor muscles during abdominal maneuvers. Arch Phys Med Rehabil 200 I; 82: 1081- 1088.

21. Tincello DG, Teare J, Fraser WD. Second trimester concentration of relaxin and pregnancy related incontinence. Eur J Obstet Gynecol Reprod Biol 2003; 106:237-238.

22. Sultan AH, Kamm MA, Hudson CN. Pudendal nerve damage during labour: prospective study before and after childbirth. Br J Obstet Gynecol 1994; 101:22-28.

23. Pessina F, Matteucci G, Esposito L, Goreffi B, Valoti M, Sgaragli G. Protection of intrinsic nerves of guinea-pig detrusor strips a anoxia/ glucopenia and reperfusion injury by taurine. Adv Exp Med Biol 2000; 483:325-333.

24. Morkved S, Bo K, Schei B, Salvesen KA. Pelvic floor muscle training during pregnancy to prevent urinary incontinence: a single-blind randomized controlled trial. Obstet Gynecol 2003; 101:313-319.

Artigo recebido: 18/02/2005

Aceito para publicação: 16/1 I/2005 